\title{
Localizing Periodicity in Time Series and Videos
}

Giorgos Karvounas ${ }^{1,2}$

gkarv@ics.forth.gr

lason Oikonomidis ${ }^{1}$

oikonom@ics.forth.gr

Antonis A. Argyros ${ }^{1,3}$

argyros@ics.forth.gr
${ }^{1}$ Institute of Computer Science, FORTH, Heraklion, Crete, Greece

${ }^{2}$ TEl of Crete, Department of Informatics Engineering, Greece

${ }^{3}$ Computer Science Department, University of Crete, Greece
Periodic patterns and motions are ubiquitous in both natural and man-made environments. Several well established tools and techniques such as the Fourier Transform [2] can be used to analyse purely periodic signals. However, in many real life scenarios, periodic signals appear as segments of larger signals containing nonperiodic parts. The detection and characterization of such periodic parts is an interesting problem that is not yet fully addressed.

In this work we propose a method that, given a time series representing a periodic signal that has a non-periodic prefix and tail, estimates the start, end and period of the periodic part of the signal. The resulting method has a small number of free parameters, is unsupervised and can detect short periodic events occurring in the context of extended non-periodic activities.

Consider as input a univariate time series $\mathbf{x}=\left\langle x_{1}, x_{2}, \ldots, x_{N}\right\rangle$. Assuming that this time series is periodic between times $b$ and $e$ and that the period of that part of the signal is $l$, our goal is to estimate $b, e$ and $l$. We formulate this task as an optimization problem in a search space defined by $b, e$ and $l$. A candidate triplet $(b, e, l)$ defines $n=\lfloor(e-b) / l\rfloor$ segments: $s_{i}=\left\langle x_{b+l \cdot(i-1)}, \ldots, x_{b+l \cdot i-1}\right\rangle, i \in\{1 \ldots n\}$. We quantify the total dissimilarity of these segments as the mean squared error among all pairs of segments: $\varepsilon_{S}(l)=\frac{1}{n \cdot l} \sum_{i=1}^{n} \sum_{j=i+1}^{n}\left\|\mathrm{~s}_{\mathrm{i}}-\mathrm{s}_{\mathrm{j}}\right\|_{2}^{2}$, where $\|\cdot\|_{2}^{2}$ denotes the squared $L_{2}$ norm. Based on this quantity, we formulate an appropriate objective function that is optimized using Particle Swarm Optimization (PSO) [1]. PSO is a stochastic method that iteratively improves a candidate solution with regard to a given measure of quality.

The core of the proposed framework is a method that, given a univariate time series containing a periodic part, detects the start, the end and the period length of that part. In practice, several phenomena can be more effectively represented as multivariate time series. We consider
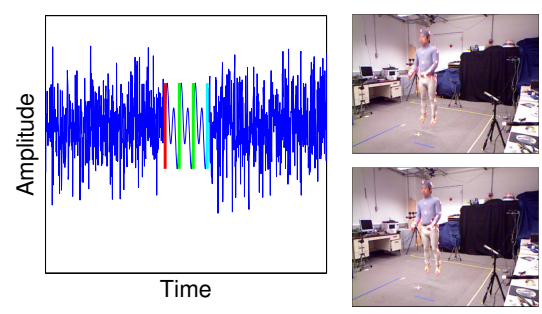

Figure 1: Left: the proposed method aims to find a periodic part within a larger, non-periodic signal. Right: indicative result on a video. Our method detects the start, the end and the period of the periodic motion (jumping). Top frame: The highest point of the jump, begin of an estimated period. Bottom frame: The highest point of the next jump, begin of next period.

multivariate time series as a set of synchronized, univariate time series. We apply the core periodicity detection method to each of them. Then, we employ a simple yet effective voting method to aggregate partial results towards characterizing the periodicity of the event that is represented with the multivariate time series.

We present the results we obtained using the proposed method. We first evaluate the performance of the method on synthetically generated sequences, determining appropriate parameters for PSO. Given these parameters, we evaluate the performance of the method under the presence of varying amounts of noise. Finally, we present results in real-world data. Specifically, we present results on detecting periodic activities using motion capture or video data as input.

[1] Maurice Clerc and James Kennedy. The particle swarm-explosion, stability, and convergence in a multidimensional complex space. Evolutionary Computation, IEEE Transactions on, 6(1):58-73, 2002.

[2] Jean Baptiste Joseph Fourier. The analytical theory of heat. The University Press, 1878. 